

\title{
Análisis contable para dinamizar la información exógena DIAN de las empresas del sector pecuario
}

\author{
Estefanía Herrera Gutiérrez ${ }^{\mathrm{a}}$ \\ Rogelio de Jesús Cárdenas Londoño ${ }^{b}$
}

\begin{abstract}
Información del artículo
Recibido: 01/06/2020

Aceptado: 30/10/2020

Clasificación JEL

E62
\end{abstract}

Este es un artículo Open Access bajo la licencia CC BY NC [https://creativecommons.org/licenses/by-nc/4.0]

\section{Enlace DOI}

https://doi.org/10.24142/rvc.n22a8

Sugerencia de citación

- Gutiérrez, E. y Cárdenas, R. (2020). Análisis contable para dinamizar la información exógena DIAN de las empresas del sector pecuario. Revista Visión Contable, 22, 158 - 177. https://doi.org/10.24142/rvc.n22a8
An Accounting Analysis to Boost DIAN Exogenous Information from Companies in the Livestock Sector

\section{Resumen \\ Abstract}

En el presente trabajo se pretende analizar el tipo de estrategias, técnicas y métodos que permiten el análisis contable de las empresas del sector pecuario y que ayudan a dinamizar la información exógena DIAN en el territorio antioqueño, partiendo de cuestionar los tipos de estrategias, técnicas y métodos que hacen o viabilizan el análisis contable en el sector de referencia. Del mismo modo, se caracteriza en el artículo la gestión que soporta el análisis contable y financiero en el campo de las empresas del sector pecuario antioqueño y colombiano a partir de la importancia relativa en términos cuantitativos, en tanto que dichos activos biológicos no se pueden dejar sin medir ni gestionar, habida cuenta de su importante contribución a la creación de valor en la consulta contable como proceso de apoyo a la empresa colombiana. Los distintos estudios emprendidos a la fecha han puesto de manifiesto que para incrementar los análisis contables y financieros corresponde mejorar la información sobre activos biológicos, dado que la que tradicionalmente ha formado parte de la información financiera publicada por las empresas infravalora dichos activos biológicos, con lo cual quedan ocultos valores importantes asociados a ellos.

\section{Palabras clave}

Análisis contable; Información exógena; Medios magnéticos; Sector pecuario; Terceros y retenciones.
This paper aims to analyze the type of strategies, techniques and methods that allow accounting analysis of companies in the livestock sector and that help to stimulate DIAN exogenous information in the Department of Antioquia, by questioning the types of strategies, techniques and methods that make or enable accounting analysis in the reference sector. In the same way, the management that the accounting and financial analysis undergoes regarding companies in the Antioquia and Colombian livestock sector is defined, based on the relative importance in quantitative terms: Those biological assets cannot be left without measuring or managing, given its contribution to the creation of value in accounting consulting as a process to support the Colombian company. The different studies undertaken to date have shown that, in order to increase accounting and financial analyzes, information on biological assets must be improved, since what traditionally has been part of the financial information published by companies underestimates these biological assets, thus important values associated with them remain hidden.

\section{Keywords}

Accounting analysis; Exogenous information; Magnetic media; Livestock sector; Third parties and withholdings.

a Correo electrónico: estefaniaherrera93@gmail.com

b Correo electrónico: rcardenas@correo.iue.edu.co 


\section{Introducción}

La investigación que dio lugar a este artículo pretendió analizar los estados financieros, entendidos como un proceso crítico dirigido a evaluar las diferentes estrategias, técnicas y métodos que están en uso -los cuales entran a valorar la información exógena que sirve para posicionar financieramente a las empresas del sector pecuario antioqueño-, que vislumbró de forma positiva las operaciones objeto de este proceso investigativo y permitió establecer mejores estimaciones y predicciones sobre las condiciones que son objeto de estudio y de análisis en este texto.

De la misma manera, este proceso investigativo se justificó en torno al papel de las nuevas empresas que se vienen creando en el territorio colombiano y, de modo específico, en la región antioqueña, sobre todo en un sector tan particular como el pecuario. Este último funciona como una entidad social que permite la asesoría y acompañamiento de las universidades y sus entes de apoyo. Además, utiliza de forma intensiva el conocimiento, a fin de responder a las expectativas de una sociedad informatizada, con mayor capacidad de participación en las decisiones sobre los modelos de producción, la organización del trabajo y la construcción de consenso sobre el futuro del desarrollo económico y social del país y sus regiones.

Hacer uso del análisis y la interpretación de los estados financieros para una joven en formación contable representa un gran reto personal y profesional, en tanto lleva a poner en práctica los conocimientos adquiridos en el desarrollo del plan de estudios en el programa de Contaduría Pública de la Institución Universitaria de Envigado (IUE). Esto conlleva hacer un análisis de las distintas fuentes de información exógena; apropiar los estados contables de las empresas del sector objeto de estudio -pecuarias-; y obtener más conocimiento con base en esa información para proyectar en función económica y financiera y prever ciertos escenarios de riesgos para las empresas del sector de interés.

Lo anterior sirve como contexto y fundamento para sostener que el tema objeto de estudio ocupa una postura hegemónica en el sector de la contabilidad, en tanto se percibe y se vive el pago de buenos salarios, aunque pocos contadores gustan de trabajar en este proceso. Sin embargo, todas las empresas deben hacerlo, por lo que se convierte en un punto de referencia laboral para los recién graduados; además, amplía la visión del sector pecuario sobre la información que rota por medio de clientes y proveedores. Se trata de un sector de la economía que, si bien extenso, lleva 
su contabilidad de modo muy informal: esto es, mueve mucho dinero en Colombia, pero con poco control. Por tanto, procesos como la presentación de información exógena no están debidamente establecidos.

En el mismo sentido, es útil el conocimiento sobre el tema por la complejidad que normalmente muestra ante la presentación de medios magnéticos. Los beneficiarios son las empresas y las personas naturales obligadas a presentar información exógena ante la Dirección de Impuestos y Aduanas Nacionales; de ahí que contadores y analistas contables, quienes se encargan de llevar estos procesos a cabo, los viabilizan en términos de mayores niveles de eficiencia. Es novedoso para el sector pecuario tener establecidas las diferentes técnicas y procedimientos de la información exógena, e ir más allá con propuestas financieras desde el análisis contable de esta información; asimismo, esto impacta en el quehacer de la gerencia de las empresas del sector agrícola, en tanto estas pueden obtener una visión del negocio mediante la información exógena e identificar los movimientos con los distintos clientes y proveedores, hecho que contribuye a una toma de decisiones ágil y oportuna.

\section{Metodología}

Este proceso se asume como un estudio de caso, entendido como una forma del conocimiento individual en la cual se privilegia la explicación que se desprende del caso mismo (Yin, 1994). De igual forma, implicó un estudio en profundidad de los casos considerados a partir del análisis contable y financiero en las empresas del sector pecuario de Antioquia. Aunque algunas veces se lo denomina "método", el estudio de caso es más un enfoque investigativo, dado que se buscó reconstruir y analizar un caso desde múltiples perspectivas que brindó la gestión del análisis contable y financiero.

El proceso de análisis fue tipo cualitativo, con enfoque crítico social. Para ello se utilizó el método analítico sintético: este orientó la gestión del análisis contable y financiero a partir del quehacer de tres empresas del sector pecuario antioqueño que, por desempeño laboral, fueron asequibles a la información que se requirió; y permitieron estructurar el caso (objeto de investigación del estudio) e identificar las características básicas de cada una de las empresas del sector pecuario que se soportan en las guías básicas de la gestión del análisis contable y financiero (categorías de análisis). Posteriormente se hizo una comparación con el fin de identificar los puntos 
comunes y las diferencias (presencias y ausencias diagnósticas contables y financieras). En consonancia, se hizo uso del enfoque mixto cuantitativo en el procesamiento de los datos y el análisis estadístico y cualitativo en la descripción de las características del objeto de investigación, el análisis contable y financiero como práctica de gestión.

Se asumió el alcance correlacional a través de un ejercicio comparativo entre lo descrito en el análisis contable y financiero en las empresas del sector pecuario en referencia. Igualmente, el diseño no experimental (ex post facto) permitió trabajar con información existente en las empresas del sector en referencia; se hizo uso de los centros de documentación a los que se tuvo acceso de forma virtual (unidades de información); y, en la misma medida, se obtuvo sustento de una investigación documental (estado del arte) para rastrear los antecedentes bibliográficos y empíricos, de acuerdo con la concepción de Hoyos (2000).

- Unidad de análisis: gestión del análisis contable y financiero a partir de la práctica desarrollada en las empresas del sector pecuario antioqueño.

- Caso: empresa del sector lácteo de Antioquia, a través de los indicadores de razones financieras, de liquidez (capital neto de trabajo, índice de solvencia, prueba ácida, rotación de inventarios, rotación de cartera, rotación de cuentas por pagar), de endeudamiento (razón de endeudamiento, razón pasivo capital), de rentabilidad (margen bruto de utilidad, margen de utilidades operacionales, margen neto de utilidades, rotación de activos, rendimiento de la inversión, rendimiento del capital común, utilidad por acción) y de cobertura (cobertura total del pasivo, razón de cobertura total).

Con base en los indicadores de razones de gestión financiera se obtuvo la información contable, administrativa y financiera requerida. Esta resume los estados financieros de tal manera que puedan interpretarse y analizarse, y comprender con ello el estado actual de la empresa en referencia al generar dicha información: todos, en conjunto, dan cuenta de la estructura financiera de la empresa del caso (Sandoval, 2018). 


\section{Marco de referencia}

Se realizó una compilación breve y precisa de conceptos y teorías que guardan relación directa con el proceso de investigación emprendido.

\section{Marco geográfico}

La empresa del sector pecuario antioqueño que sirve de referencia tiene asiento en el Municipio de San Pedro de los Milagros, en el norte del departamento de Antioquia. Esta población se caracteriza porque su especialización laboral se centra en la producción en empresas lácteas, situación que se confirma por eventos como las Fiestas de la Leche y, en general, por todos los derivados de este producto que allí se procesan. En tanto manipulan un producto altamente perecedero, las plantas industriales que producen estos alimentos deben vigilarlos y analizarlos correctamente durante todos los pasos de la cadena de frío hasta su destino final: el consumidor.

La demanda local, regional, nacional e internacional de productos lácteos ha experimentado un crecimiento considerable en años recientes, situación que ha llevado a la industria a superar retos técnicos y tecnológicos importantes.

\section{Marco teórico}

Para dar cuenta de este apartado se recurre al análisis de los componentes y partes de los eslabones de la cadena de frío. Esta se emplea en el abastecimiento y distribución de los lácteos, sus productos y derivados. En esta medida, tales actividades deben conservar y mantener la cadena del frío en intervalos técnicos parametrizados para el sector alimentario ( 2 a 8 grados centígrados). Sin embargo, las temperaturas dependen en gran medida del tipo de producto abastecido en la cadena y de los requisitos del fabricante.

Preservar la cadena de frío en el transporte (lácteos sin fermentación y con fermentación) es un proceso delicado que requiere materiales y asesoría técnica de los expertos en el ramo; y de modo fundamental, se hace más evidente y se expresa en la salvaguarda de la salud del consumidor final, que valida este momento de verdad.

La preservación de la cadena de frío, basada en las buenas prácticas y en la inversión en aislamiento, suponen un importante rubro que se refleja en aho- 
rros de producción, transporte, almacenamiento y venta. Los productos lácteos - casi todos deben ser conservados rigurosamente en frío- ostentan grados, capacidades y necesidades de conservación distintos; y la capacidad de conservación afecta por igual su calidad y seguridad. Por regla general, los métodos de conservación empleados en la industria láctea se centran en la pasteurización (control bacteriano), el control de la temperatura (control de procesos enzimáticos mediante la vigilancia de la cadena de frío) y el diseño del tipo de envases (control físico que garantice temperatura interior y hermeticidad).

La Secretaría de Agricultura y Desarrollo Rural del Departamento de Antioquia dejó saber, tanto en sus Lineamientos de la política de agricultura familiar campesina como en el Plan de ordenamiento territorial agropecuario, que se han invertido más de 230.000 millones de pesos a fin de ampliar capacidades institucionales en términos de producción, comercialización y tecnificación del sector agropecuario. Esto tiene impacto en más de 15.000 productores y alrededor de 1700 unidades productivas agropecuarias tecnificadas en todas las regiones del departamento (Secretaría de Agricultura y Desarrollo Rural del Departamento de Antioquia, 2020). Además, esta corporación ha prestado asistencia técnica a todos los municipios de Antioquia-como complemento a las unidades municipales de asistencia técnica agropecuaria (Umata) y las secretarías de agricultura municipales- para llegar a cada predio y mejorar las prácticas agropecuarias, con inversiones cercanas a los \$200 millones por municipio (Secretaría de Agricultura y Desarrollo Rural del Departamento de Antioquia, 2020).

Marón (2017), al igual que Menezes y Pinel (2018), refieren que los gobiernos hacen grandes apuestas financieras para incentivar las buenas prácticas agropecuarias en el sector lechero. Tal es el caso en Colombia: se han destinado \$2400 millones de pesos para hacer intervenciones directas en las firmas o empresas más representativas del sector -una de ellas, Colanta, en el territorio antiqueño-, las cuales recibirán un acompañamiento especializado que se dará por etapas, la primera de las cuales consiste en diagnosticar e implementar una metodología de escalamiento y un plan de acción a corto plazo para propender a su crecimiento.

Las empresas del sector transformador lácteo han tenido ventas superiores a las $\$ 800$ millones en el último año. A este respecto se destacan, entre otras, San Martín Lácteos en Caldas; Rp Quesos, Lácteos La Laguna y Andalucía de Inversiones en Cundinamarca; Productora de Alimentos Alina y Procesadora y Comercializadora de Alimentos Renacer en Santander; Pasteurizadora La Mejor en Norte de Santander; Lácteos del Campo 
Caqueteño y Alimentos Gamar en Caquetá; e Inversiones La Holandesa y Coocampo en Boyacá, datos que coinciden con lo establecido al respecto por Estrada (2015).

En oposición, otras empresas afrontan el gran reto de reinventarse y ampliar su portafolio, en tanto que se puede vislumbrar un panorama incierto para ellas de no seguir lo recomendado para el sector. Entre estas se encuentran Productos Alimenticios Rinconcito en Cauca; Indasan en Risaralda; Industria Quindiana de Lácteos y Alimentos Limitada - Quinalac, Cafequipe en Quindío; Agrolácteos Potosí en Nariño; Procelácteos en Huila; Derivados Lácteos del Llano, y La Catira Industria Láctea en el Meta; Industrias Alimenticias Pinar del Río, Inversiones Espejo Askar y El Jarro Productos Alimenticios en el Depto. de Atlántico; Wellness Food Company, Alimentos Nebraska, Derivados Lácteos El Pino, Productos Montana, Derilac, Lácteos Buenavista, Distribuciones Lácteos Oriente, Lácteos Río Grande, Quesitos Maya y El Llano en Antioquia; y Lácteos Campo Real, Productos Lácteos Paco’s, País Emprendedor y Lácteos Mileto en Bogotá.

\section{Marco legal o jurídico del sector agropecuario}

El marco legal y jurídico para el sector agropecuario que enmarca este proceso investigativo tiene origen en Ley 89 de 1993, por la cual se estableció la cuota de Fomento Ganadero y Lechero y se creó el Fondo Nacional del Ganado. A esta la siguieron, en orden descendente, la Ley 1659 de 2013, mediante la que se creó el sistema nacional de identificación, información y trazabilidad animal; la Ley 1375 de 2009, por la cual se establecieron las tasas por la prestación de servicios a través del sistema nacional de identificación y de información del ganado bovino (Sinigán); la Ley 1372 de 2009, por medio de la cual se aprobó el Acuerdo de libre comercio entre la República de Colombia y los Estados de la Asociación Europea de Libre Comercio (AELC); y la Ley 1133 de 2007, por medio de la cual se creó e implementó el programa Agro Ingreso Seguro -AIS.

En consonancia con lo dicho, el Decreto 957 del 5 junio de 2019 estableció la nueva clasificación de las empresas según los ingresos brutos anuales por actividades ordinarias; se determinaron con esto tres sectores a evaluarse para identificar si una organización es micro, pequeña, mediana o gran empresa en Colombia. La tabla 1 resume esta clasificación. 
TABLA 1. Clasificación de las empresas según el Decreto 957 de 2019

\begin{tabular}{|c|c|c|c|c|}
\hline \multicolumn{5}{|c|}{ Sector manufacturero } \\
\hline \multicolumn{5}{|c|}{ Rango de ingresos UVT } \\
\hline \multirow[t]{2}{*}{ Clasificación } & \multicolumn{2}{|c|}{ Rango de ingresos UVT } & \multicolumn{2}{|c|}{ Rango de ingresos (\$) } \\
\hline & Mayor que & Menor o igual que & Mayor que & Menor o igual \\
\hline Microempresa & - & 23.563 & 0 & 781.254 .828 \\
\hline Pequeña empresa & 23.563 & 204.995 & 781.254 .828 & 6.796 .814 .220 \\
\hline Mediana empresa & 204.995 & 1.736 .565 & 6.796 .814 .220 & 57.577 .549 .140 \\
\hline Gran empresa & 1.736 .565 & - & 57.577 .549 .140 & - \\
\hline \multicolumn{5}{|c|}{ Sector servicios } \\
\hline & Mayor que & Menor o igual que & Mayor que & Menor o igual \\
\hline Microempresa & - & 32.988 & & 1.093 .750 .128 \\
\hline Pequeña empresa & 32.988 & 131.951 & 1.093 .750 .128 & 4.374.967.356 \\
\hline Mediana empresa & 131.951 & 483.034 & 4.374.967.356 & 16.015 .475 .304 \\
\hline Gran empresa & 483.034 & & 16.015 .475 .304 & - \\
\hline \multicolumn{5}{|c|}{ Sector comercio } \\
\hline & Mayor que & Menor o igual que & Mayor que & Menor o igual \\
\hline Microempresa & - & 44.769 & & 1.484 .360 .964 \\
\hline Pequeña empresa & 44.769 & 431.196 & 1.484 .360 .964 & 14.296 .734 .576 \\
\hline Mediana empresa & 431.196 & 2.160 .692 & 14.296 .734 .576 & 71.639 .903 .952 \\
\hline Gran empresa & 2.160 .692 & & 71.639.903.952 & - \\
\hline
\end{tabular}

En lo que atañe al caso que nos ocupa, y como se ha dicho, la organización analizada es de tipo agropecuario y su objeto principal se encuentra en su razón social. El análisis de sus actividades de compra y venta, así como de su producido, permiten ubicarla en el rango de mediana empresa.

En Colombia, según el informe Dinámica de Creación de Empresas de la Confederación Colombiana de Cámaras de comercio - Confecámaras (2019), elaborado con base en la información del Registro Único Empresarial y Social - RUES de las 57 Cámaras de Comercio del país, se indica que

Durante el primer trimestre del 2019 en Colombia fueron creadas 96.914 unidas productivas, un aumento del $9,7 \%$ frente al mismo periodo del año 2018. Según el informe del total de las empresas registradas el 
$77,8 \%$ corresponde a personas naturales y el $22,2 \%$ a sociedades, en ambas obteniendo un aumento de aproximadamente $10 \%$ con respecto al año anterior. (Confecámaras, 2019).

99,6 \% de las empresas creadas en el primer trimestre del año en cuestión se consideran microempresas; y $0,3 \%$, pequeñas empresas. Se presentó, así mismo, una dinámica positiva de creación de empresas en el país: aumentó 9,7\% durante el primer trimestre de 2019.

\section{Marco conceptual y red de categorías}

Para Ortiz (2017), un análisis financiero es un conjunto de técnicas utilizadas para diagnosticar la situación y perspectivas de la empresa con el fin de tomar decisiones adecuadas. Este proceso comprende la recopilación, interpretación, comparación y estudio de los estados financieros y datos operacionales de un negocio (Ortiz, 2017). En el análisis se toman en cuenta los históricos de una empresa y se refleja una situación contable y financiera en una fecha definida; por lo general, se realiza para la terminación de un periodo determinado.

El análisis financiero se realiza a partir de la información contable, a través de indicadores y razones financieras y de acuerdo con tres métodos:

- Análisis vertical: también conocido como análisis estático, puesto que solo se toma un estado (estado de situación financiera y estado de resultados). En este se analizan los datos de las subpartidas contables frente a la proporción que esta representa de la cuenta mayor. En este análisis el tiempo no es una variable determinante; se utilizan los porcentajes para dichas relaciones.

- Análisis horizontal: es un análisis comparativo, también reconocido por ser dinámico. El tiempo es un factor importante, puesto que se analizan las partidas de los estados financieros en diferentes fechas o cortes. Así mismo, se analizan las variaciones (positivas o negativas) que presentan las cuentas de un periodo frente a otros. Este tipo de análisis también permite mostrar el desarrollo o reducción que ha tenido el negocio. Su resultado se expresa en variaciones, traducibles en gráficos que facilitan una interpretación más analítica de la situación.

- Análisis proyectado: se utiliza para analizar presupuestos o estados financieros proyectados. 
Para el caso de estudio se emplearon los métodos vertical y horizontal, que hicieron posible conocer el estado de la organización de manera contable y financiera para los cortes de fin de año, al tiempo que ayudaron a identificar las proporciones más representativas de las subcuentas frente a las cuentas principales ${ }^{1}$. Una vez cumplida esta fase, se procedió a utilizar una herramienta financiera conocida como indicadores financieros.

\subsection{Razones financieras orientadoras}

Las razones financieras que orientaron este proceso investigativo son indicadores utilizados para medir o cuantificar la realidad económica y financiera de la empresa en referencia, al igual que su capacidad de asumir las obligaciones que se le encargaron para desarrollar su objeto social en función de los requerimientos del proceso investigativo emprendido (Uriarte, 2006).

En línea con la elección de los métodos de análisis se emplearon razones estáticas y dinámicas, así: las primeras se utilizaron para el informe del estado de la situación financiera (balance); mientras que las segundas se aplicaron a los informes de estado de resultados. En algunos casos se pueden combinar, con lo que se da origen a razones mixtas. Existen, entonces, razones de cuatro tipos, corroborables con lo dicho por Olivos (2012) y Cuervo et al. (2013).

- De liquidez

- De endeudamiento o de administración de deudas

- De actividad

- De rentabilidad

\subsection{Marco contextual}

El presente análisis toma como recursos los estados financieros de una empresa cuyo objeto social es la producción, importación, exportación y comercialización de toda clase de productos e insumos de uso agrícola y pecuario. Como se dijo, desarrolla sus actividades en el municipio de San Pedro de los Milagros (Antioquia): las condiciones de accesibilidad a los

En este sentido, se acopió la información de los tres últimos años (2017-2019) para tener más conocimiento del comportamiento de la organización durante sus gestiones más recientes. 
servicios públicos y vías de acceso se dificultan en esta población, hecho que da lugar a incrementos en sus costos operacionales. Fue creada en 2005 y su sede administrativa se encuentra en la ciudad de Medellín. La empresa cuenta con una planta de personal de aproximadamente 15 empleados cuyo rango salarial no supera dos salarios mínimos, salvo los encargados administrativos y técnicos de la empresa.

Las actividades de producción agropecuaria de la empresa corresponden a la explotación de un hato lechero, esto es, cría y engorde de semoviente en el área de la ganadería. Las actividades comenzaron en 2010. La empresa se cataloga como "en desarrollo" puesto que sus primeras transacciones económicas y financieras solo dieron réditos desde 2017, momento a partir del cual se escalonaron anualmente para ver los resultados financieros satisfactorios; a la fecha, esta planta requerirá siete años para que su potencial máximo de producción se alcance (además, se considera que debe cumplir con unos estándares técnicos prefigurados).

El hato se dedica de forma exclusiva a la cría, puesto que las condiciones tropicales y climáticas de la zona donde se ubica hacen imposible cualquier otro tipo de labor (ceba o engorde). La población estimada del hato oscila entre 900 y 1200 cabezas de ganado. La carne en pie es vendida en la finca; por ende, su valor comercial para la venta disminuye su precio en un $5 \mathrm{o}$ $10 \%$ del valor razonable.

\section{Resultadlos orientadores y derivaciones}

La realización del proceso de investigación descrito arrojó variables relevantes a tener en cuenta en el cumplimiento de lo propuesto. De ello se deriva que se haya optado por realizar una síntesis de carácter descriptivo, ya que este se determinó como el tipo de estudio que pretende obtener información acerca del estado actual de los fenómenos.

Naturalmente, recabar toda la información posible acerca de un fenómeno pareció, en principio, una meta de difícil alcance. No obstante, de acuerdo con los propósitos de los métodos de estudio en ejecución, se determinó cuáles fueron los factores o variables cuya situación se identificó. La descripción pretendió incluir aspectos cualitativos y cuantitativos del fenómeno objeto de estudio; esto es, trascendió de la mera acumulación de datos a un proceso de análisis e interpretación compuesto con base en un marco teórico (Moreno, 2000) 
La composición de tal estudio descriptivo, de base cuantitativa, correspondió a un análisis integral del estado de situación financiera y estado de resultados de la organización objeto de estudio durante sus últimos tres años de funcionamiento: se establecieron las cuentas más relevantes y se determinaron las que presentaron mayor decrecimiento en el periodo evaluado para establecer qué motivó tales alteraciones. Se tuvieron en cuenta las notas a los estados financieros, las políticas contables y demás información financiera que se consideró pertinente para el desarrollo del análisis.

La realización de las labores nombradas encontró soporte en el método de investigación inductivo: es decir, se obtuvieron conclusiones generales a partir de premisas particulares (Samaja, 2005). Se trata del método científico más usual, en el que pueden distinguirse cuatro pasos esenciales: observación de los hechos para su registro; clasificación y estudio de estos hechos; derivación inductiva que parte de los hechos, que permite llegar a una generalización; y contrastación a partir de la unidad de análisis.

\subsection{Principales resultados obtenidos}

Se presentan a continuación (tabla 2) los estados financieros que se analizaron con el objeto de determinar posibles estrategias que ayuden al mejoramiento de la empresa; como se expuso, se analizaron los tres últimos años de funcionamiento: 2017-2019. Los estados fueron elaborados según las Normas Internacionales de Información Financiera para pequeñas y medianas empresas (NIIF para las pymes), sector al cual pertenece la organización objeto de estudio. 
TABLA 2. Estado de situación financiera, expresado en pesos colombianos, de Inversiones Mario Velásquez

\begin{tabular}{|c|c|c|c|}
\hline \multicolumn{4}{|c|}{ Activos } \\
\hline & 2019 & 2018 & 2017 \\
\hline Efectivo y equivalentes de efectivo & 13.409 & 430.410 & 449.016 \\
\hline Deudores comerciales y otras cuentas por cobrar & 97.566 & 84.928 & 78.885 \\
\hline Inventario & 3.723 & 27.282 & \\
\hline Otros activos no financieros & 667 & 2046 & 1123 \\
\hline Total activo corriente & 115.365 & 563.079 & 529.024 \\
\hline Propiedades, planta y equipo & 465.643 & 524.888 & 589.819 \\
\hline Activos biológicos ganadería & 949.245 & 1.050 .629 & 921.951 \\
\hline Activos biológicos & 2.199 .106 & 7.010 .453 & 6.364 .592 \\
\hline Activos por impuestos diferidos & & 243.424 & 378.329 \\
\hline Total activo no corriente & 3.613 .993 & 8.829 .394 & 8.254 .691 \\
\hline Total activos & 3.729 .358 & 9.392 .472 & 8.783 .715 \\
\hline \multicolumn{4}{|c|}{ Pasivos } \\
\hline & 2019 & 2018 & 2017 \\
\hline $\begin{array}{l}\text { Cuentas corrientes comerciales por pagar y otras } \\
\text { cuentas por pagar }\end{array}$ & 102.638 & 69.957 & 52.419 \\
\hline Pasivos por impuestos corrientes & 39.305 & 40.375 & 36.541 \\
\hline Beneficios a empleados & 25.264 & 30.023 & 21.804 \\
\hline Total pasivo corriente & 167.208 & 140.355 & 110.763 \\
\hline $\begin{array}{l}\text { Cuentas corrientes comerciales por pagar y otras } \\
\text { cuentas por pagar }\end{array}$ & 5.240 .796 & 4.992 .048 & 4.208 .381 \\
\hline Pasivo por impuesto diferido & & 326.291 & 139.978 \\
\hline Total pasivo no corriente & 5.240 .796 & 5.318 .339 & 4.348 .359 \\
\hline Total pasivo & 5.408 .003 & 5.458 .694 & 4.459 .122 \\
\hline \multicolumn{4}{|c|}{ Patrimonio } \\
\hline & 2019 & 2018 & 2017 \\
\hline Capital suscrito y pagado & 5.100 .000 & 5.100 .000 & 5.100 .000 \\
\hline Reservas acumuladas & & - & 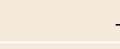 \\
\hline Resultados acumulados & -1.790 .859 & -1.351 .812 & -1.114 .363 \\
\hline Resultado del ejercicio & -5.612 .423 & -439.048 & -237.577 \\
\hline Otro resultado integral (ORI) & 360.652 & 360.652 & 312.419 \\
\hline Utilidades retenidas en adopción por primera vez & 263.986 & 263.986 & 264.115 \\
\hline Total patrimonio & -1.678 .645 & 3.933 .778 & 4.324 .594 \\
\hline Total pasivo y patrimonio & 3.729 .358 & 9.392 .472 & 8.783 .715 \\
\hline Fuente: elaboración propia & & & \\
\hline
\end{tabular}




\begin{tabular}{|l|r|r|r|}
\hline TABLA 3. Estado de resultados integral, expresado en pesos colombianos \\
\hline Operaciones continuas & \multicolumn{1}{|c|}{$\mathbf{2 0 1 9}$} & \multicolumn{1}{|c|}{$\mathbf{2 0 1 8}$} & \multicolumn{1}{|c|}{$\mathbf{2 0 1 7}$} \\
\hline Ingresos de actividades ordinarias costo de ventas & 295.971 & 126.778 & 187.228 \\
\hline Utilidad bruta & -297350 & -124.088 & -169.342 \\
\hline Otros ingresos & -1380 & 2690 & 17.886 \\
\hline Gastos de administración & 3146 & 7212 & 8497 \\
\hline Gastos de operación & -140.225 & -40.983 & -38.178 \\
\hline Deterioro activos biológicos & -660.196 & - & - \\
\hline Otros gastos & -4.782 .183 & - & - \\
\hline Resultado de actividades de operación & -34.213 & -8881 & -12.823 \\
\hline Ingreso financiero & -5.615 .050 & -39.962 & -24.618 \\
\hline Costo financiero & 4901 & 10.225 & 12.318 \\
\hline Costo financiero neto & -46.660 & -49.157 & -134.011 \\
\hline Utilidad antes de impuesto & -41.760 & -38.932 & -121.693 \\
\hline Impuesto de renta corriente & -5.656 .810 & -78.894 & -146.311 \\
\hline Impuesto CREE corriente & -38.481 & -38.935 & -25.473 \\
\hline Impuesto diferido & - & - & -9171 \\
\hline Ganancia (pérdida) del año antes del ORI & 82.868 & -321219 & -56.622 \\
\hline Otro resultado integral & -5.612 .423 & -439.048 & -237.577 \\
\hline Ingreso por valor razonable & & & \\
\hline Activos biológicos & -5.612 .423 & -390.816 & 55.769 \\
\hline Total otro resultado integral del año & & & \\
\hline Ganancia (pérdida) del año & & & \\
\hline Fuente: elaboración propia & & & \\
\hline
\end{tabular}

[COLOMBIA]

Respecto de la categoría activos corrientes, 2018 la empresa internamente en sus activos reclasificó 9,7 hectáreas al cultivo de los insumos necesarios para su emprendimiento; igualmente, la política contable de la empresa la clasifica al interior de sus inventarios. En 2019, la empresa definió que el material destinado a sus labores productivas se usaría para el análisis de mercado, para así ajustar el inventario; el análisis se determinó en función del deterioro que sufren las materias primas que están en relación de la cadena productiva de la misma, y se estima en un $10 \%$ mensual sobre el valor del inventario.

El incremento evidenciado entre el año 2017 al 2018 en la cuenta de otros activos no financieros se debe a los pagos realizados por anticipado a varios proveedores. La variación en la cuenta de efectivo, por su parte, se 
debe a que el movimiento realizado dentro de la cuenta fiduciaria del banco de preferencia según se evidencia en las notas a los estados financieros. De igual forma, si se toma la categoría activos no corrientes, la variación entre los años 2019 y 2018 en los activos biológicos de la cadena productiva se debe a que, por la disminución considerable de los precios de los lácteos, la administración de la empresa decidió realizar un deterioro en los cultivos del $68 \%$, ya que los costos incurridos en la producción no suplían los bajos precios del mercado.

$\mathrm{Al}$ hacer uso de manera racional de las NIIF, los cambios en las cuentas más significativas correspondieron a la posibilidad de revaluación de cualquiera de los de su clase de propiedad, planta y equipo, y los activos biológicos. Por otro lado, si se retoma la cuenta de pasivos se visualiza un aumento de los costos y gastos por pagar, al igual que la cuenta de proveedores nacionales y las deudas que posee la empresa con los directos de la misma, razón por la cual esta cuenta se encuentra en niveles superiores o elevada. Este proceso llevó a las directivas de la empresa a ajustar las políticas contables y estipular que los préstamos a los socios no causaran intereses (Grajales y Montoya, 2017).

En relación con la categoría patrimonio se evidencia que el capital suscrito y pagado se mantuvo durante los tres años evaluados. En 2018 y 2019 la empresa generó pérdida en el ejercicio contable debido a que los ingresos percibidos no solventaron los costos y gastos del desarrollo de su razón social y quehacer. Este precedente permitió evaluar el estado de resultados, que confirma lo expuesto .

En esta misma línea del análisis se evidenció una reclasificación de las cuentas contables que causó un incremento significativo en los gastos; ello se debe al cumplimiento de la Norma Internacional, la cual establece que el costo del mantenimiento se debe separar en lo ocurrido antes y después de que la plantación esté en condiciones de producción, y establecerlo como gasto en los estados financieros.

La empresa estudiada ha presentado pérdidas recurrentes entre $2017 \mathrm{y}$ 2019. Esta situación se torna cada vez más crítica por cuanto en este último año, como consecuencia del deterioro aplicado a la cadena de producción y el estado actual del mercado de los lácteos, se presenta un escenario desfavorable para la producción y comercialización de estos productos: en la actualidad, los precios de compra no son los más competitivos frente al costo de producción. 
Con la identificación de las cuentas de mayor variación se evaluaron las siguientes razones financieras:

- Liquidez: durante 2017 y 2018, la empresa mantuvo una liquidez sólida; pero en 2019 disminuyó de forma significativa, debido a los ajustes realizados por el deterioro del activo biológico (la modificación se debe al cambio solicitado por la gerencia de la empresa).

- Capital de trabajo: este rubro es alto y posiblemente refleja que el dinero se encontraba sin movimiento en 2017 y 2018; en cambio, en 2019 el indicador presentó una disminución considerable debido a un incremento en sus costos y gastos por pagar.

- Inventario: en 2019 presentó un deterioro, lo cual lleva a que el activo corriente presente un detrimento importante.

- Prueba ácida: los resultados de la prueba ácida evidencian problemas de liquidez en 2019 con un índice de 0,67, inferior al mínimo requerido (1).

- Endeudamiento: en función de la liquidez, la empresa manejo sus deudas de una forma poco conservadora: comprometía el $50 \%$ de sus activos. En el año 2019 se muestra que el endeudamiento es astronómico, por lo cual debe ser objeto de intervención inmediata.

- Actividad: el indicador más significativo en las razones de actividad es la rotación de inventario, puesto que tiene un aumento y este obedece a la reclasificación que hizo la empresa de sus gastos llevándolos al costo y el deterioro del inventario que se estableció durante el año 2019.

- Cuentas por pagar: a través de ellas se evidenció que la empresa realiza pagos a sus proveedores antes de tener retorno de sus ventas (ingreso del efectivo), razón por la cual la liquidez y el ciclo de operación de la empresa se ven afectados de forma directa.

- Rentabilidad: la actividad principal de la empresa se basa en la producción y la cadena de valor de los lácteos se clasifica como "en desarrollo". Esto quiere decir que aún no ha logrado su máxima producción y, por ende, los ingresos que genera son mínimos. Por lo tanto, las ventas que se evidencian en los estados financieros corresponden, en su mayoría, a la venta de estos productos. Esta no es suficiente para cubrir sus costos y gastos de funcionamiento, hecho que daría lugar a índices negativos de rentabilidad. Esta situación no es del total agrado de los socios de la empresa porque, según notas de los estados financieros, tienen un capital muy representativo en ello. 
Es importante anotar que cada las categorías en uso permitieron realizar el análisis del tipo de estrategias, técnicas y métodos que posibilitan, a su turno, el análisis contable de las empresas del sector pecuario y ayudan a dinamizar la información exógena DIAN en el territorio antioqueño.

\section{Recomendaciones}

Con base en razones financieras, y visto el análisis mixto de los datos de los estados financieros de los años de referencia, se presentan las siguientes recomendaciones o encargos:

- Corresponde verificar los gastos administrativos, en tanto son significativos dentro de los gastos totales de la operación. Se recomienda o sugiere corroborar que todos los gastos en los que se incurra durante el periodo sean necesarios para la operación, con el fin de minimizar una proporción de estos.

- Es pertinente utilizar el método de flujo de caja futuro traído a valor presente para validar si las inversiones realizadas a la fecha y futuras serán compensadas.

- En vista del déficit continúo en los resultados del ejercicio contable y financiero de la empresa estudiada, y con la finalidad de salvarla de entrar en proceso de liquidación o disolución, se aconseja tomar en cuenta lo expuesto en el numeral 2 del artículo 457 del Código de comercio: allí se establece que una sociedad entrará en proceso de disolución cuando las pérdidas reduzcan el patrimonio neto por debajo del $50 \%$ del capital suscrito.

- Se sugiere capitalizar acciones, entendido esto último como un proceso mediante el cual se establezcan asociaciones con organizaciones que dispongan de los recursos de la empresa estudiada para la producción y explotación del ciclo lácteo, de tal suerte que esta última se encargue de la comercialización de sus productos e incurra con ello en menores costos y gastos.

- Vista la disminución significativa en los resultados operativos de la empresa en el territorio antioqueño, se recomienda replantear la idea del negocio y redirigir su razón de ser a la producción alternativa, la cual ostenta mayores ventajas competitivas en la región donde esta se ubica. 


\section{Conclusiones}

Con los análisis vertical y horizontal de los estados financieros se identificó que las partidas más significativas en cuanto a las variaciones -en detrimento o aumento- fueron efectivos y equivalentes en función de las categorías de referencia (efectivos, inventario, activo biológico, cuentas corrientes por pagar a corto y largo plazo).

Al revisar el estado de resultados, los cambios más representativos se presentaron en las cuentas de ingresos, costos, gastos y resultados de los ejercicios contables y financieros, que indicaron una disminución de $1,178 \%$. De la misma forma, el análisis de los indicadores financieros permite afirmar que durante 2019 se presentó una disminución significativa en los indicadores de liquidez y endeudamiento debido a la reducción de los activos biológicos por deterioro, lo que afecta de modo directo el activo evaluado con los pasivos. En cuanto a los indicadores de actividad, se evidenció que la empresa está generando un ciclo de operación que se contrapone a lo usual -pago anticipado a sus proveedores, antes de tener el pago de sus clientes-, en función de generar mayores niveles de confianza y fidelidad. En la misma línea, al sopesar los resultados de los indicadores de rentabilidad se percibe una sensación de desaliento para los inversionistas: para 2019, todos los resultados fueron negativos debido a que los ingresos por las actividades de desarrollo del ciclo lácteo no cubrieron los gastos y costos, hecho que causó pérdidas en el ejercicio contable y financiero.

$\mathrm{Al}$ sector lácteo y pecuario de Antioquia se le presentó la gran oportunidad de adoptar las NIIF para pymes. En el caso de la empresa estudiada, lo anterior dio paso a una reclasificación significativa en las cuentas de activos, costos y gastos que determinaron un detrimento en el resultado del ejercicio del último año evaluado; de no tenerse en cuenta las acciones descritas en el artículo 459 del Código de Comercio Nacional, esto llevará a una situación de alta complejidad o de posible cierre.

Lo descrito, explorado y explicado en el presente trabajo permite reflexionar en función de los negocios que se llevan a cabo en el sector pecuario y agrícola del territorio antioqueño: sean de tardío o de corto rendimiento, generan utilidad a largo plazo. Por esto, los grupos de interés, y en particular, los inversionistas, deberán tener muy claro que la tasa retorno de la inversión asumida no se presentará de forma inmediata; en esta medida, la generación de utilidades sobre recursos invertidos demanda realizar el ciclo completo de producción, transformación y comercialización para ver plasmado el gran sueño. 


\section{Referencias}

Cuervo, J., Osorio, J., \& Duque, M. (2013). Costeo basado en actividades ABC: Gestión basada en actividades ABM. Segunda Edición (Segunda). Ecoe Ediciones.

Estrada, R. (2015). Plan estratégico agroindustrial de calidad y ambiental, del sector pecuario de Córdoba, con base en las BPA, BPM y BPG. Universidad de Manizales, 27. http:// ridum.umanizales.edu.co:8080/ xmlui/handle/6789/1992.

Grajales, L. y Montoya, M. (2017). Impacto financiero que genera el manejo del inventario de activos biológicos y productos agrícolas en el sector pecuario, bajo Normas Internacionales, NIIF para Pymes: Análisis en actividad de gallinas ponedoras. [Tesis de pregrado, Universidad del Valle]. https://pdfs.semanticscholar.org/8cae/8c38654b5f72210d054cbeeb9c93c6773303.pdf? $\mathrm{ga}=2.83963737 .1505704052 .159$ 1340907-668554084.1591340907.

Hoyos, C. (2000). Un modelo para investigación documental: Guía teórico-práctica sobre construcción de estados del arte con importantes reflexiones sobre la investigación (Primera Edición). Señal Editora.

Marón, G. (2017). Diseño de un sistema de costeo para una empresa pecuaria Pedro Marón S.A. [Tesis de pregrado, Instituto Universitario Aero- náutico]. https://rdu.iua.edu.ar/bitstream/123456789/1225/1/PG-Mar\%c3\%b3n\%20Gabriela\%20Anabel.pdf.

Menezes, G. y Pinel, M. (2010). Auditoría social en empresa brasileña transnacional del sector pecuario que actúa en Amazonia legal. Axe II, Symposium 5. Estudio de caso. Independencias Dependencias - Interdependencias, VI Congreso CEISAL 2010, Toulouse. https://halshs.archives-ouvertes.fr/halshs-00502812/fr/.

Moreno, M. (2000). Introducción a la metodología de la investigación educativa II. Editorial Progreso. https://books.google.com.co/books?i$\mathrm{d}=15 \mathrm{t}$ h 9 QddksC\&pg=PA128\&d$\mathrm{q}=$ estudio+descriptivo+en+la+investigacion $\&$ hl $=\mathrm{es}-419 \& \mathrm{sa}=\mathrm{X} \& \mathrm{ve}-$ d=0ahUKEwjBpuTT0- .

Olivos, L. (2012). Perspectiva Para El sector agropecuario colombiano desde la experiencia chilena en Normas Internacionales De Información Financiera: Activos Biológicos [Tesis de pregrado, Universidad San Buenaventura]. http://bibliotecadigital.usb.edu.co/bitstream/10819/994/1/Perspectiva_sector_agropecuario_Olivos_2012.pdf.

Ortiz, H. (2017). Finanzas básicas para no financieros. Cengage Learning. https://www.academia.edu/32924786/ FINANZAS_B\%C3\%81SICAS_para_ no_financieros_Segunda_edici\%C3\%B3n. 
Samaja, J. (2005). Epistemología y Metodología: Elementos para una teoría de la investigación científica. (Tercera Edición). EUDEBA. https://bdigital. uncu.edu.ar/objetos_digitales/3383/ fischetticuyo06-23.pdf.

Sandoval, C. (2018). El sector pecuario y la tecnificación fiscal. Las tribulaciones de un productor. Análisis Plural, 11. https://rei.iteso.mx/bitstream/ handle/11117/5355/E4.El+sector+pecuario $+y+$ la+tecnificacio $\% 20$ $\mathrm{n}+$ fiscal.pdf? sequence $=2$.

Secretaría de Agricultura y Desarrollo Rural del Departamento de Antioquia. (2020). Informe de gestión. Se- cretaría de Agricultura y Desarrollo Rural del Departamento de Antioquia. https://www.antioquiahonesta. com/secretarias/secretaria-de-agricultura-y-desarrollo-rural/.

Uriarte, F. (2006). Introducción a las Normas Internacionales de Información Financiera. Marcial Pons.

Yin, R. (1994). Case Study Research: Design and Methods (Vol. 5). Sage Publications. https://books.google. com.co/books/about/Case_study_ research.html?id=AvYOAQAAMAAJ\&redir_esc $=y \cdot g a=2.8396$ 3737.1505704052.1591340907668554084.1591340907. 